\title{
Relationships of oxygen uptake, heart rate, and ratings of perceived exertion in persons with paraplegia during functional neuromuscular stimulation assisted ambulation
}

\author{
Patrick L Jacobs, K John Klose, Rosalind Guest, Belinda Needham-Shropshire, James G Broton and Barth A Green \\ The Miami Project to Cure Paralysis and the Department of Neurological Surgery, University of Miami School of \\ Medicine, Miami, Florida 33136, USA
}

\begin{abstract}
Previous reports have described significant limitations in the daily use of functional neuromuscular stimulation (FNS) ambulation systems by persons with spinal cord injuries (SCI). The potential application of these devices to provide physiological benefits as an exercise modality has prompted a reconsideration of the technology. However, the acute physiological effects related to the use of FNS systems have not been thoroughly examined. The purpose of this study was to investigate the relationships of oxygen consumpation $\left(\mathrm{VO}_{2}\right)$, heart rate (HR), and ratings of perceived exertion (RPE) during FNS ambulation by persons with SCI paraplegia. Eleven persons with thoracic level paraplegia, aged 21.5 to 38.0 years, participated in an incremental FNS ambulation test. Metabolic measures were collected continuously via open circuit spirometry as the subjects performed a series of ambulation passes of progressively increasing pace. At the end of each ambulation pass, HR and RPE measures were collected. The test was terminated when either the subjects judged the effort to be maximal or when the investigators deemed the effort to be maximal based on HR. A strong linear relationship was documented between the $\mathrm{VO}_{2}$ and $\mathrm{HR}$ measures of all subjects throughout subpeak levels of FNS ambulation. RPE did not vary proportionally with $\mathrm{VO}_{2}$ until relatively high levels of exercise intensity were reached. This study indicates that HR, but not RPE, is an appropriate indicator of exercise intensity for persons with SCI paraplegia using a FNS ambulation system.
\end{abstract}

Keywords: spinal cord injury; paraplegia; functional neuromuscular stimulation; ambulation; rate of perceived exertion

\section{Introduction}

Functional neuromuscular stimulation (FNS) assisted ambulation systems have been demonstrated to be a feasible method to enable certain persons with complete spinal cord injuries (SCI) to stand and ambulate. While limitations in the daily use of such devices for functional mobility have been reported, ${ }^{1-4}$ the application of these systems for exercise conditioning purposes has not been studied extensively. The relatively high energy expenditures associated with these systems as well as the ability to utilize the relatively larger lower extremity musculature may indicate the potential for cardiovascular benefits. However, valid measures of exercise intensity are required in order to quantify exertion levels.

Exercise intensity, for diagnostic or exercise prescription purposes, has been expressed in terms of oxygen consumption $\left(\mathrm{VO}_{2}\right)$, heart rate $(\mathrm{HR})$, and/or ratings of perceived exertion (RPE). ${ }^{5-8}$ Maximal

Correspondence: PL Jacobs oxygen consumption $\left(\mathrm{VO}_{2 \max }\right)$ is generally accepted as the criterion measure of cardiorespiratory capacity. ${ }^{5}$ Accordingly, recommended intensity levels for particular purposes may be accurately expressed in terms of $\mathrm{VO}_{2 \max }$. However, use of this measure is generally restricted to sophisticated research settings due to the specialized equipment required. Field studies and training programs commonly utilize either relative HR (\%pkHR) or RPE measures as valid indicators of intensity, as both measures have been demonstrated to display linear relationships with relative $\mathrm{VO}_{2}$ $\left(\% \mathrm{pkVO} \mathrm{VO}_{2}\right)$ at increasing submaximal workloads during upper and lower extremity exercise in nondisabled populations.9,10 Bar-On and Nene demonstrated that persons with SCI levels below T4 display a linear $\mathrm{HR}-\mathrm{VO}_{2}$ relationship during incremental arm ergometry. ${ }^{11}$ The standard $\%$ pkHR $-\% \mathrm{pkVO}_{2}$ association for non-disabled persons has also been confirmed to be applicable to persons with paraplegia during arm exercise. ${ }^{12}$ RPE has been established to be a valid marker of exercise intensity for persons with SCI and other wheelchair bound populations during 
arm ergometry. ${ }^{13,14}$ Numerous researchers have used RPE as a measure of intensity during arm exercise testing and training studies involving persons with paraplegia. ${ }^{15-17}$

Investigations of FNS ambulation systems have primarily dealt with hardware and control system issues. Few studies of FNS ambulation devices, without external knee or hip bracing, have examined the physiological effects of such systems. ${ }^{18-21}$ Those that have reported physiological measures presented data on a limited number of subjects (one to four) and focused the emphasis on gait efficiency or the energy cost associated with one particular self-selected ambulation pace.

The associations of $\mathrm{VO}_{2}, \mathrm{HR}$, and RPE may exhibit unique variations in persons with SCI utilizing FNS devices to drive paralyzed limbs. Persons with paraplegia exhibit altered hemodynamic responses during arm ergometry as compared with non-disabled controls. ${ }^{22-26}$ Decreased levels of venous return limit stroke volume and result in increased heart rates at given workloads. It is not known how venous return may be affected during FNS ambulation or how variations in hemodynamic responses to the FNS ambulation situation might impact the $\mathrm{HR}-\mathrm{VO}_{2}$ relationship.

The 15 point RPE scale (Figure 1) was developed by Borg as a simple tool that varied directly with HR through a submaximal range of exercise. ${ }^{7}$ Subsequent research efforts indicated that RPE values are based on a combination of inputs from active muscles and/or joints (peripheral cues) and signals from general cardiopulmonary input (central cues). ${ }^{27}$ During low to moderate levels of exertion, RPE is related primarily to peripheral cues while the central feedback mechanisms contribute to the self reported ratings during more intense levels of effort.

Although the person with complete SCI paraplegia is devoid of direct peripheral feedback from the exercising musculature, therapists have utilized RPE measures during FNS ambulation training. However, the validity of this type of self rating scale in this setting had not been sufficiently determined. The purpose of this study was to examine the relationships between $\mathrm{VO}_{2}, \mathrm{HR}$, and RPE displayed in persons with paraplegia during a FNS ambulation test. The results of this study may indicate if HR and/or RPE are valid indicators of exercise intensity in this setting.

\section{Methods}

\section{Subjects}

Eleven persons with SCI (T4-T11) volunteered to participate in this study (Male, 10; Female, 1). The subjects' ages ranged from 21.5 to 38.0 years $(27.78 \pm 5.64)$ and the time since the traumatic injury ranged from 1.1 to 9.2 years $(3.93 \pm 0.92)(\mathrm{X} \pm \mathrm{SD})$. The neurological level of injury was determined by a licensed physical therapist utilizing the ASIA Standards for Neurological Classification scale. ${ }^{28}$ All subjects

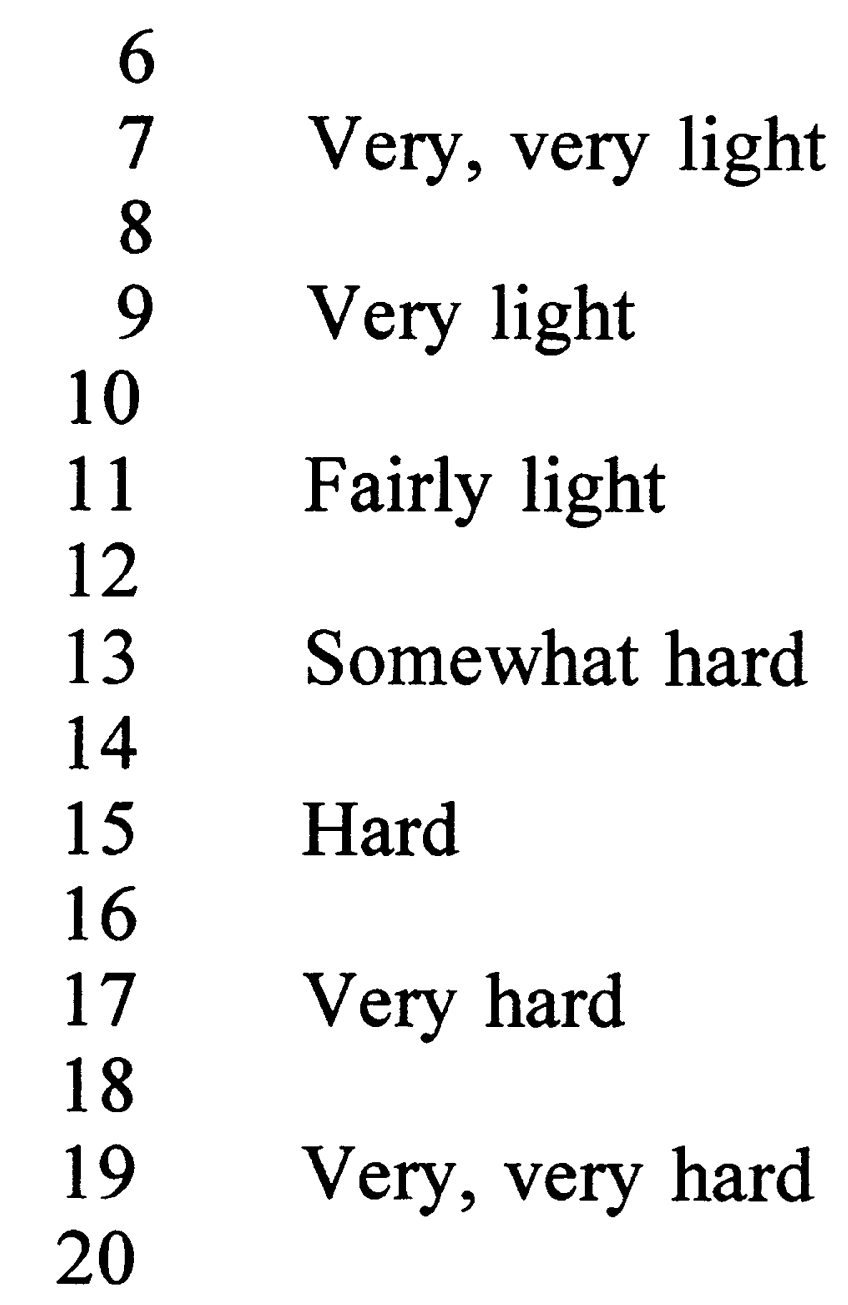

Figure 1 Borg's 15 point ratings of perceived exertion scale. Subjects were directed to indicate the level at which they

were experienced with the FNS ambulation system and had completed a minimum of 3 months of training in the laboratory immediately prior to this testing procedure. All subjects were apparently healthy individuals who were without evidence of cardiopulmonary disease and were not taking any medication that might affect test results. The testing procedures were verbally explained to the subjects and a written informed consent was obtained in accordance with the University of Miami Human Subjects Institutional Review Board.

\section{FNS device}

This study utilized the Parastep- $1^{\circledR}$ which is a commercially available FNS system produced by Sigmetics, Inc. of Northfield, Illinois. This system was designed to allow persons with SCI paraplegia to stand and ambulate limited distances. ${ }^{29}$ It utilizes a battery powered, microprocessor controlled six channel stimulator which is attached to an adapted walker frame. Six 
channels of electrical stimulation (three channels bilaterally) are applied with surface electrodes on the (1) quadriceps region, (2) gluteal region, (3) upper lateral leg near the common perioneal nerve. The standing position is established by knee extension and hip extension via muscular contractions of the quadricep and gluteal musculature respectively. The stepping action is generated via a flexor withdrawal response produced by a brief stimulation of the common peroneal nerve concurrent with cessation of stimulation to the quadriceps and gluteals of that leg. The walker frame is outfitted with a series of finger switches which allow the user to independently control the stimulation intensity and walking pace.

\section{Testing procedure}

Subjects were asked to refrain from alcohol, tobacco or caffeine ingestion for a period of $12 \mathrm{~h}$ and from food for $2 \mathrm{~h}$ prior to the testing. The subjects were weighed and asked to independently don the FNS system. Many of the subjects used a lightweight plastic insert, an ankle-foot orthosis, inside their shoes to improve the stepping action. Once the equipment was applied, the subjects stood and took a limited preparatory walk (less than $5 \mathrm{~min}$ ) in order to determine that the surface electrode placement was appropriate and that all equipment was in proper working order. The subjects were then positioned in their wheelchair at the end of the testing area where they sat quietly for a minimum of $30 \mathrm{~min}$.

During the rest period the subjects were fitted with a facemask which was connected to a SensorMedics Horizon System to allow metabolic measures via open circuit spirometry continuously throughout the experiment. Prior to each experiment, the metabolic cart was calibrated using known gas concentrations and volumes. Three minutes of resting metabolic measures were collected at the initiation of the experiment. Resting HR was obtained, via direct palpation of the carotid artery, during this period. Following the rest period, the subjects were directed to activate the FNS system and stand in place for a 1 min period. At the end of the $1 \mathrm{~min}$ period, the subjects were asked to point to the appropriate category on a 6 to 20 point Borg chart of perceived exertion while HR was also measured. The subjects were then instructed to ambulate, at a minimum pace, along a 30 foot walkway. The metabolic cart followed the subjects throughout the ambulation process. At the end of the runway, the subjects were directed to stop and again indicate the RPE level while HR was obtained. This constituted one 'pass'. Subjects were then directed to turn and again traverse the walkway, at a somewhat faster pace. Subjects continued this process, increasing the pace of ambulation slightly on each pass. The time period of each 30 foot run was measured and used to ensure that the pace was increased as directed. The test was terminated when the subjects deemed their efforts to be maximal or when the investigators determined that a point of maximal exertion had been reached. $\mathrm{VO}_{2}$ plateau, $\mathrm{HR}$, and respiratory exchange ratio were used for the determination of maximal exertion.

\section{Statistical analysis}

The $\mathrm{VO}_{2}, \mathrm{HR}$, and RPE measures served as the dependent variables of this study. The $\mathrm{VO}_{2}$ measures were determined by the last complete $15 \mathrm{~s}$ segment calculated by the Horizon system for each walking pass. The absolute $\mathrm{VO}_{2}$ and $\mathrm{HR}$ measures of each subject were adjusted into $\% \mathrm{pkVO}_{2}$ and $\%$ pkHR values. A least squares line of best fit was determined for the $\% \mathrm{pkVO}_{2}-$ $\%$ pkHR and the $\% \mathrm{pkVO}_{2}$ - RPE relationships for each subject and for the combined group data. A lack of fit $\mathrm{f}$ test was then applied to each relation to determine if the relation was linear or if a higher order equation was more appropriate. ${ }^{30} \mathrm{~A}$ piecewise least squares linear regression with breakpoint was also calculated for each relation. ${ }^{31}$ Statistical significance was accepted at the $P<0.05$ level of confidence.

\section{Results}

All subjects completed a sufficient number of walking passes to supply at least five sets of data points. Equipment malfunction of the metabolic cart resulted in incomplete sampling of metabolic measures for one subject, therefore the analyses described below are based on the remaining 10 subjects.

The oxygen uptake data of all subjects is shown in Figure 2. Oxygen consumption values tended to increase as a function of the increasing energy needs related to the increasing pace of the ambulation test. The most dramatic oxygen uptake increase took place during the initial ambulation pass. The group mean oxygen uptake value for the initial pass, $16.19 \mathrm{ml} \mathrm{kg}^{-1} \min ^{-1}$, was 4.2 times the mean of the resting $\mathrm{VO}_{2}$ values $\left(3.84 \mathrm{ml} \mathrm{kg}^{-1}\right.$ $\min ^{-1}$ ) and was approximately $70 \%$ of the mean peak $\mathrm{VO}_{2}$ value $\left(22.9 \mathrm{ml} \mathrm{kg}^{-1} \mathrm{~min}^{-1}\right)$. For comparisons with HR and RPE, the oxygen consumption values were expressed as $\% \mathrm{pkVO}_{2}$.

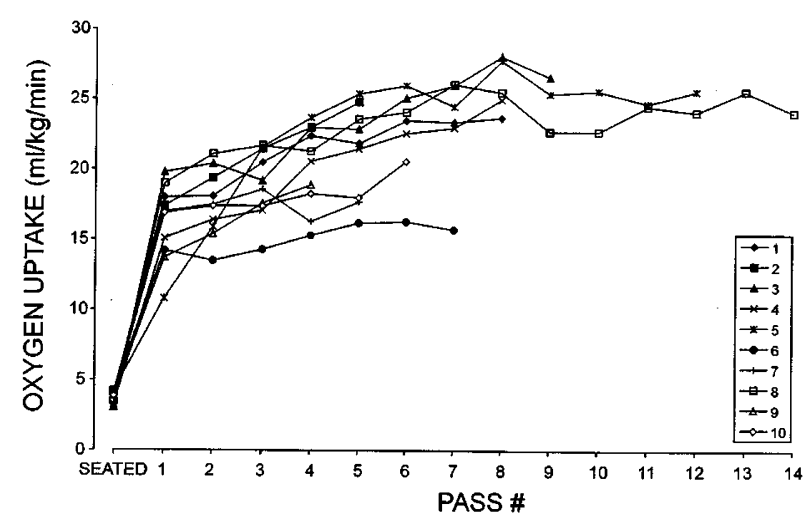

Figure 2 Oxygen uptake at each ambulation pass during an incremental FNS ambulation test 
The HR responses displayed a general increase in all subjects throughout the ambulation test. The mean peak HR was $192.2 \mathrm{bpm}(175-212)$. The mean HR value after the initial pass was $147.5 \mathrm{bpm}$, which was approximately twice the mean of the resting values $(75.0 \mathrm{bpm})$. The heart rate data were expressed as $\%$ pkHR, and were compared to $\% \mathrm{pkVO}_{2}$ values (Figure 3). A strong linear relationship was evident when data from individual subjects were analyzed, with correlation coefficients ranging from 0.777 to 0.987 (mean $=0.953 \pm 0.063)$. The correlation coefficient calculated with the grouped data was 0.924 , and the following regression equation was determined:

$$
\mathrm{y}=0.2795+0.7151 \mathrm{x}
$$

where $y=\% p k H R$ and $x=\% p V_{2}$. The statistically significant linear relationship between $\% \mathrm{pkVO}_{2}$ and $\%$ pkHR $(P<0.001)$ indicates that $\mathrm{HR}$ increased proportionally with the increasing energy needs of FNS assisted ambulation.

All subjects also displayed a general, but not consistent, increase in the RPE responses as the ambulation test progressed to peak exercise. Eight of the eleven subjects reached an exercise peak at which they judged the exertion level on the Borg scale as at least '19' ('very, very hard'). Two subjects judged their final pass as 'very hard' on the scale. ${ }^{16,17}$ One subject reached a peak RPE report of '13' ('somewhat hard'), despite a peak HR of $194 \mathrm{bpm}$.

The association of the $\% \mathrm{pkVO}_{2}$ values with the RPE responses of each subject throughout the ambulation tests is displayed in Figure 4. The initial increase in oxygen consumption described above was not matched with a substantial alteration of the RPE response for the majority of subjects. Linear regression calculations of the $\% \mathrm{pkVO}_{2}$-RPE relationships resulted in a lower coefficient of determination value for the grouped response (0.507) as well as the individual

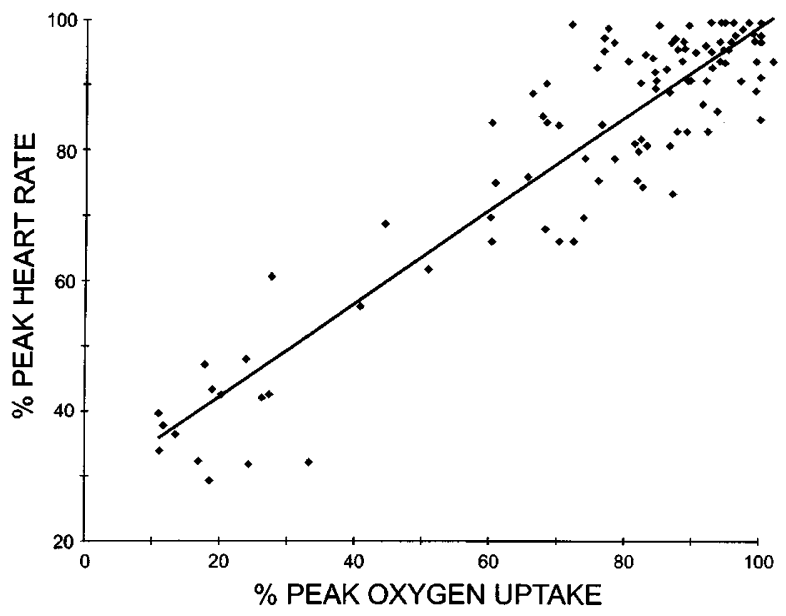

Figure 3 Relationship between oxygen uptake and heart rate

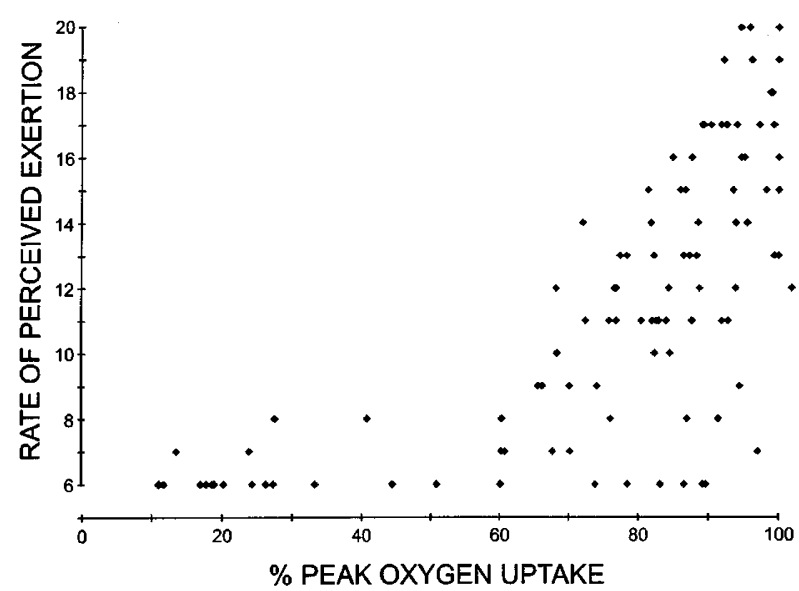

Figure 4 Relationship between oxygen uptake and ratings of perceived exertion in persons with paraplegia during FNS

subjects' responses $(0.692 \pm 0.228)$. Individual lack of fit $\mathrm{f}$-tests revealed that the $\% \mathrm{VO}_{2}$ - $\mathrm{RPE}$ relation was statistically non-linear for seven of the ten subjects and for the entire study group. The group responses were more accurately predicted $\left(r^{2}=0.606\right)$ with the following quadratic equation:

$$
\mathrm{y}=8.2594-15.294 \mathrm{x}+23.619 \mathrm{x}^{2}
$$

where $\mathrm{y}=\mathrm{RPE}$ and $\mathrm{x}=\% \mathrm{VO}_{2}$.

However, piecewise linear regression provided a statistically superior model for the prediction of RPE based on the $\% \mathrm{pkVO}_{2}$ response. Using the piecewise model, an $\mathrm{r}^{2}$ value of 0.794 was calculated for the total study group, with values calculated for the individual subjects ranging from 0.779 to 0.999 . The $\% \mathrm{pkVO}_{2}$ breakpoint for the total study group occurred at 0.755 of the $\mathrm{VO}_{2 \text { peak. }}$ A post-hoc piecewise linear regression was also calculated for the total group using RPE as the predictor of $\% \mathrm{pkHR}$. This analysis resulted in a breakpoint occurring at 0.817 of the \%pkHR which related to an absolute HR value of $157.9 \mathrm{bpm}$.

\section{Discussion}

The peak $\mathrm{VO}_{2}$ and RPE values found in this study are similar to peak values reported in various arm ergometry and wheelchair ergometry studies as performed by persons with paraplegia. ${ }^{11,12,15-17,23-27}$ However, the HR values at peak exertion were higher than values generally reported for this population during arm exercise. These findings are dissimilar to exercise responses reported for persons without disabilities. Peak arm exercise efforts of non-disabled persons generally produce significantly lower peak $\mathrm{VO}_{2}$ levels $(20-35 \%$ lower) than leg exercise despite similar peak HR levels. ${ }^{9,10}$

The heart rate and oxygen uptake responses to the initial FNS ambulation pass were similar to those 
previously reported. ${ }^{18-21}$ While subjects were directed to perform the first ambulation pass at a minimum pace, the HR values were elevated significantly from resting levels. Similarly the $\mathrm{VO}_{2}$ values increased to more than four times the resting values with this very controlled pace.

This study demonstrates that a direct linear relationship exists between $\%$ pkHR and $\% \mathrm{pkVO}_{2}$ throughout submaximal ranges of FNS ambulation performed by persons with paraplegia. Approximately $85 \%$ of the variation in $\% \mathrm{pkVO}_{2}$ could be attributed to variations in $\% \mathrm{pkHR}$. This relationship supports the notion that $\mathrm{HR}$ is a valid indicator of work intensity in this setting.

Additionally, the results of this study demonstrated a similar $\mathrm{HR}-\mathrm{VO}_{2}$ relationshiip for FNS ambulators as that established for the general population. General guidelines for fitness enhancement of the non-disabled population recommend training intensities of $60-90 \%$ pkHR which are reported to correspond with $50-85 \%$ $\mathrm{pkVO}_{2}{ }^{5}$ The least squares regression equation established in this study produces virtually the same values $\left(50-85 \% \quad \mathrm{pkVO}_{2}\right.$ relating to $62.4-88.9 \%$ pkHR) (Table 1).

Perception of physical exertion was described by Borg $^{7}$ as a combination or 'Gestalt' of signals arising from throughout the body. However, Borg's 15 point RPE scale was developed in the quest of a simple tool that would vary directly with HR. The numbers used to delineate the intervals on the scale were selected to correspond with approximately one tenth of the HR values. This RPE scale has been previously demonstrated to be a reliable device within a variety of healthy and clinical populations.

Ekblom and Golgbarg ${ }^{27}$ were the first investigators to distinguish between central and peripheral cues in regards to perception of effort sense. In this context, 'central' refers to systemic physiological cues such as cardiopulmonary signals (HR, respiration rate, minute ventilation) or blood lactate levels. 'Peripheral' refers to feedback from the local exercising musculature such as cues of touch, pressure, temperature or proprioception. It is generally accepted that peripheral cues dominate the reference field of RPE during low to moderate levels of exertion. Central factors significantly contribute to perception of effort as exercise intensity increases.

Table 1 Values of \%pkHR and RPE established with data from this study at given values of $\% \mathrm{pkVO}_{2}$

\begin{tabular}{lcc}
\hline & $50 \% \mathrm{pkVO}_{2}$ & $85 \% \mathrm{pkVO}_{2}$ \\
\hline$\% \mathrm{pkHR}$ & $62.4 \pm 5.4 \%$ & $88.9 \pm 5.8 \%$ \\
& $(60 \%)$ & $(90 \%)$ \\
$\mathrm{RPE}$ & $6-8$ & $11.9 \pm 3.2$ \\
& $(12-13)$ & $(16)$ \\
\hline
\end{tabular}

Data in parenthesis represent values previously established for the general non-disabled population
Conscious monitoring is required for accurate self estimation of exertion levels. Without conscious awareness there are no cues (direct or indirect) from which to determine exertion. ${ }^{38}$ Persons with complete paraplegia are devoid of sensory cues directly derived from artificially contracted paralyzed musculature. Therefore, at relatively low to moderate levels of exertion the FNS ambulator is severely limited in the amount and types of conscious internal cues normally available to non-disabled persons during exercise. Persons responsible for FNS training have assumed that without the overriding peripheral signals, FNS ambulators would become more attuned to central cues. This rationale follows the theory that other physically challenged individuals augment missing perception by relying more heavily on other input (eg, blind persons become more attuned to auditory and proprioceptive cues). However, the results of this study indicate that persons with SCI are unable to accurately estimate their level of exertion in this situation until quite high levels of intensity are reached. Several subjects were noted to report effort perceptions of 'very, very, easy' at exertion levels of $70-90 \% \mathrm{pkVO}_{2}$ (Figure 4).

While this study did not attempt to discriminate between the RPE responses based on proficiency of FNS ambulation, the authors did observe that there was an association between the RPE responses and mastery of this system. Subjects who were able to use the FNS system adeptly and place less body weight on their arms were noted to exhibit significantly less accurate RPE responses than less competent subjects. It would seem that the upper extremity work required by the less proficient users provided increased feedback for perception of effort. Additional research is needed to quantify the possible relationships between the degree of leg versus arm work and the RPE responses of FNS ambulators.

Use of self-selected pacing by FNS ambulators may result in walking at inappropriately high exercise intensities. Without kinesthetic awareness of the exercising musculature, the principle feedback mechanism may be visual, with the emphasis of attention on walking pace. The pace of FNS ambulation is considerably slower than the pace of non-disabled walking due to the nature of the system itself. Lacking accurate feedback, the FNS user may attempt to increase the pace of ambulation past suitable levels of intensity. Chronic training at inappropriately high intensities or volumes has been associated with an overtraining state, which has been related to reduced exercise benefits and ultimately decreased health levels. ${ }^{33-36}$ Decreased functional status, inherent in many persons with paraplegia, increases the risk of overtraining.

Exercise intensity is a critical factor in the assessment of functional status and in the development of appropriate exercise prescriptions. This study indicates that HR remains a useful index of exercise intensity in this unique setting. However, levels of 
intensity or duration appropriate for efficient safe training effects have not been established. Persons with decreased functional status are reported to benefit from initial exercise programs with intensity levels equivalent to $40-50 \%$ of $\mathrm{pkVO}_{2}{ }^{5}$ Such intensity levels were displayed by several subjects during the stationary FNS standing phase. Therefore, additional research is required regarding a more precise utilization of the linear relationship of $\mathrm{HR}-\mathrm{VO}_{2}$ in exercise recommendations for persons with paraplegia using FNS assisted ambulation systems.

\section{Conclusions}

This study suggests that RPE is not a valid indicator of intensity of effort for persons with paraplegia using a FNS ambulation system. These findings corroborated the two factor model of RPE as originally suggested by Elblom and Goldbarg. ${ }^{27}$ That model attributes the physiological input of RPE primarily to peripheral muscular cues during low to moderate intensity levels of exertion while central cardiopulmonary cues contribute to effort awareness during more intense efforts. Persons with SCI paraplegia are devoid of direct feedback from musculature below the point of injury and are therefore unable to accurately assess work effort levels during FNS ambulation. Such a dissociation of the normal HR-RPE and $\mathrm{VO}_{2}-\mathrm{RPE}$ relationships may also be present with other populations displaying diminished feedback from the exercising musculature. Therefore, the use of RPE in such populations should be examined prior to widespread use. HR was confirmed to be an appropriate indicator of exercise intensity in this setting and therefore is an acceptable measure for use in field and experimental settings. Additional research is recommended to establish appropriate levels of exercise intensity and duration for optimal physiological benefits.

\section{References}

1 Gallien $\mathrm{P}$ et al. Restoration of gait by functional electrical stimulation for spinal cord injured patients. Paraplegia 1995; 33: $660-664$.

2 Kantor $\mathrm{C}$ et al. Report on a conference on motor prostheses for workplace mobility of paraplegic patients in North America. Paraplegia 1993; 31: 439-456.

3 Stallard J, Major RE, Patrick JH. A review of the fundamental design problems of providing ambulation for paraplegic patients. Paraplegia 1989; 27: $70-75$.

4 Yarkony GM, Roth EJ, Cybulski G, Jaeger RJ. Neuromuscular stimulation in spinal cord injury: restoration of functional movement of extremities. Arch Phys Med Rehabil 1992; 73: $78-86$.

5 American College of Sports Medicine. Guidelines for exercise testing and prescription. 4th ed. Lea \& Febiger: Philadelphia. 1991: pp 93-109.

6 Pollack ML. Wilmore JH. Exercise in health and disease. evaluation and prescription for preventionand rehabilitation. 2nd ed. W.B. Saunders: Philadelphia. 1990: pp 100-123.

7 Borg G. Perceived exertion as an indicator of somatic stress. Scand J Rehabil Med 1970; 2: 92 - 98.
8 Nobel BJ. Clinical applications of perceived exertion. Med Sci Sports Exer 1982; 14: 406-411.

9 Franklin BA. Exercise testing, training, and arm ergometry. Sports Med 1985; 2: 100-119.

10 Franklin BA. Aerobic exercise training programs for the upper body. Med Sci Sports Exerc 1989; 21: S141-S148.

11 Bar-On ZH, Nene AV. Relationship between heart rate and oxygen uptake in thoracic level paraplegics. Paraplegia 1990; 28: $87-95$.

12 Hooker SP et al. Oxygen uptake and heart rate relationship in persons with spinal cord injury. Med Sci Sports Exerc 1993; 25: $1115-1119$.

13 Birk CA, Birk TJ, Gavron S, Hackett C. Relationship of cardiopulmonary parameters with perceived exertion in wheelchair users. (Abstract) Med Sci Sports Exerc 1985; 17: 250.

14 Birk TJ, Birk CA. Use of ratings of perceived exertion for exercise prescription. Sports Med 1987; 4: 1-8.

15 Bhambhani YN, Eriksson P, Steadward RD. Reliability of peak physiological responses during wheelchair ergometry in persons with spinal cord injury. Arch Phys Med Rehabil 1991; 72: 559562.

16 Franklin BF et al. Field test estimation of maximal oxygen consumption in wheelchair users. Arch Phys Med Rehabil 1990; 71: $574-578$

17 Martel G, Noreau L, Jobin J. Physiological responses to maximal exercise on arm cranking and wheelchair ergometry with paraplegics. Paraplegia 1991; 29: $447-456$.

18 Isakorov E et al. Energy cost and physiological reactions to effort during activation of paraplegics by functional electrical stimulation. Scan J Rehab Med Suppl 1985; 12: $102-107$.

19 Marsolais EB, Edwards BG. Energy costs of walking and standing with functional neuromuscular stimulation and long leg braces. Arch Phys Med Rehabil 1988; 69: 243-249.

20 Miller P, Kobetic R, Lew R. Energy costs of walking and standing using functional electrical stimulation. Proc 13th Association for the Advancement of Rehabilitation and Assistive Technologies Ann Conf. Washington 1990: pp 155-156.

21 Winchester P, Carollo JJ, Habasevich R. Physiologic costs of reciprocal gait in FES assisted walking. Paraplegia 1994; 32: $680-686$.

22 DeBruin MI, Binkhorst RA. Cardiac output of paraplegics during exercise. Int J Sports Med 1984; 5: 175-176.

23 Hjentnes N. Oxygen uptake and cardiac output in graded arm exercise in paraplegics with low level spinal injuries. Scand $J$ Rehabil Med 1977; 9: $107-113$.

24 Hopman MTE, Oeseburg B, Binkhorst RA. Cardiovascular responses in paraplegic subjects during arm exercise. Eur J Appl Phsyiol 1992; 65: $73-78$.

25 Jehl JL et al. Cardiac output during exercise in paraplegic subjects. Eur J Appl Physiol 1991; 62: 256-260.

26 Van Loan M, McCluer S, Loftin JM, Boileau RA. Comparisons of physiological responses to maximal arm exercise among ablebodied, paraplegics and quadriplegics. Paraplegia 1987; 25: 397 405.

27 Ekblom B, Goldbarg AN. The influence of physical training and other factors on subjective rating of perceived exertion. Acta Physiol Scand 1971; 83: 399-406.

28 American Spinal Injuries Association. Standards for the neurological classification of spinal injury patients. ASIA, Chicago. 1996.

29 Graupe D, Kohn KH. Functional electrical stimulation for ambulation by paraplegics. Krieger: Malabar F1, 1994: pp 1-4.

30 Kleinbaum DG, Kupper LL, Muller KE. Applied regression analysis and other multivariable methods. 2nd ed. Duxbury: Belmont, CA, 1988: pp 231-248.

31 CSS: Statistica (Volume II)., StatSoft, Inc., Tulsa, OK. 1991: pp $423-462$.

32 Edwards RHT et al. Physiological correlates of perceived exertion in continuous and intermittent exercise with the same average power output. Eur J Clin Invest 1972; 2: $108-$ 114.

33 Brown RL et al. Overtraining of athletes; a round table. Phys and Sportsmed 1983; 11: $93-110$. 
Relationships of $\mathrm{VO}_{2}$, HR and RPE during FNS ambulation

PL Jacobs et al

34 Dressendorpher RH, Wade CE. The muscular overuse syndrome in long-distance runners. Phys and Sportsmed 1983; 11: 116-130.

35 Kuipers H, Keizer HA. Overtraining in elite athletes. Sports Med 1988; 6: $79-92$
36 Lehmann M, Foster C, Keul J. Overtraining in endurance athletes: a brief review. Med Sci Sport Exer 1993; 25: 854-862. 